\title{
The ALMA Spectroscopic Survey in the Hubble Ultra Deep Field
}

DOI:

10.3847/1538-4357/833/1/73

\section{Document Version}

Final published version

Link to publication record in Manchester Research Explorer

\section{Citation for published version (APA):}

Carilli, C. L., Chluba, J., Decarli, R., Walter, F., Aravena, M., Wagg, J., Popping, G., Cortes, P., Hodge, J., \& Weiss, A. (2016). The ALMA Spectroscopic Survey in the Hubble Ultra Deep Field: Implications for Spectral Line Intensity Mapping at Millimeter Wavelengths and CMB Spectral Distortions. The Astrophysical Journal, 833(1). https://doi.org/10.3847/1538-4357/833/1/73

Published in:

The Astrophysical Journal

\section{Citing this paper}

Please note that where the full-text provided on Manchester Research Explorer is the Author Accepted Manuscript or Proof version this may differ from the final Published version. If citing, it is advised that you check and use the publisher's definitive version.

\section{General rights}

Copyright and moral rights for the publications made accessible in the Research Explorer are retained by the authors and/or other copyright owners and it is a condition of accessing publications that users recognise and abide by the legal requirements associated with these rights.

\section{Takedown policy}

If you believe that this document breaches copyright please refer to the University of Manchester's Takedown Procedures [http://man.ac.uk/04Y6Bo] or contact uml.scholarlycommunications@manchester.ac.uk providing relevant details, so we can investigate your claim.

\section{OPEN ACCESS}


THE ALMA SPECTROSCOPIC SURVEY IN THE HUBBLE ULTRA DEEP FIELD: IMPLICATIONS FOR SPECTRAL LINE INTENSITY MAPPING AT MILLIMETER WAVELENGTHS AND CMB SPECTRAL DISTORTIONS

\author{
C. L. Carilli ${ }^{1,2}$, J. Chluba ${ }^{3}$, R. Decarlit ${ }^{4}$, F. Walter ${ }^{1,4}$, M. Aravena ${ }^{5}$, J. WagG $^{6}$, G. Popping ${ }^{7}$, P. Cortes ${ }^{8,9}$, J. Hodge $^{10}$, \\ A. Weiss ${ }^{11}$, F. Bertoldi ${ }^{12}$, AND D. RieChers ${ }^{13}$ \\ ${ }^{1}$ National Radio Astronomy Observatory, P.O. Box 0, Socorro, NM 87801, USA; ccarilli@aoc.nrao.edu \\ ${ }_{3}^{2}$ Astrophysics Group, Cavendish Laboratory, JJ Thomson Avenue, Cambridge CB3 0HE, UK \\ 3 Jodrell Bank Centre for Astrophysics, University of Manchester, Oxford Road, M13 9PL, UK \\ ${ }^{4}$ Max-Planck Institute for Astronomy, D-69117 Heidelberg, Germany \\ ${ }^{5}$ Nucleo de Astronomia, Facultad de Ingenieria, Universidad Diego Portales, Av. Ejercito 441, Santiago, Chile \\ ${ }^{6}$ Square Kilometre Array Organisation, Lower Withington, Cheshire, UK \\ ${ }^{7}$ European Southern Observatory, Karl-Schwarzschild-Strasse 2, D-85748, Garching, Germany \\ ${ }^{8}$ Joint ALMA Observatory-ESO, Av. Alonso de Cordova, 3104, Santiago, Chile \\ ${ }^{9}$ National Radio Astronomy Observatory, 520 Edgemont Rd, Charlottesville, VA, 22903, USA \\ ${ }^{10}$ Leiden Observatory, Leiden University, Niels Bohrweg 2, NL2333 RA Leiden, The Netherlands \\ ${ }^{11}$ Max-Planck-Institut für Radioastronomie, Auf dem Hügel 69, D-53121 Bonn, Germany \\ ${ }^{12}$ Argelander Institute for Astronomy, University of Bonn, Auf dem Hügel 71, D-53121 Bonn, Germany \\ ${ }^{13}$ Cornell University, 220 Space Sciences Building, Ithaca, NY 14853, USA \\ Received 2016 May 6; revised 2016 August 9; accepted 2016 August 11; published 2016 December 8
}

\begin{abstract}
We present direct estimates of the mean sky brightness temperature in observing bands around 99 and $242 \mathrm{GHz}$ due to line emission from distant galaxies. These values are calculated from the summed line emission observed in a blind, deep survey for spectral line emission from high redshift galaxies using ALMA (the ALMA spectral deep field observations "ASPECS" survey). In the $99 \mathrm{GHz}$ band, the mean brightness will be dominated by rotational transitions of CO from intermediate and high redshift galaxies. In the $242 \mathrm{GHz}$ band, the emission could be a combination of higher order $\mathrm{CO}$ lines, and possibly [C II] $158 \mu \mathrm{m}$ line emission from very high redshift galaxies $(z \sim 6-7)$. The mean line surface brightness is a quantity that is relevant to measurements of spectral distortions of the cosmic microwave background, and as a potential tool for studying large-scale structures in the early universe using intensity mapping. While the cosmic volume and the number of detections are admittedly small, this pilot survey provides a direct measure of the mean line surface brightness, independent of conversion factors, excitation, or other galaxy formation model assumptions. The mean surface brightness in the $99 \mathrm{GHZ}$ band is: $T_{B}=0.94 \pm 0.09 \mu \mathrm{K}$. In the $242 \mathrm{GHz}$ band, the mean brightness is: $T_{B}=0.55 \pm 0.033 \mu \mathrm{K}$. These should be interpreted as lower limits on the average sky signal, since we only include lines detected individually in the blind survey, while in a low resolution intensity mapping experiment, there will also be the summed contribution from lower luminosity galaxies that cannot be detected individually in the current blind survey.
\end{abstract}

Key words: cosmic background radiation - dark ages, reionization, first stars - galaxies: formation - molecular data - radio lines: general - techniques: imaging spectroscopy

\section{INTRODUCTION}

Intensity mapping of the cumulative $\mathrm{CO}$ and other millimeter and submillimeter line emission from early galaxies has been proposed as a new means to probe very large-scale structures in the distant universe (Carilli 2011; Gong et al. 2011, 2012; Yue et al. 2015). Intensity mapping entails low spatial and spectral resolution imaging of the sky to obtain the mean brightness due to the cumulative emission from myriad discrete cosmic sources. While interferometric arrays like ALMA, the JVLA, and NOEMA, can detect CO and [C II] $158 \mu \mathrm{m}$ (and in cases of high luminosity sources, other lines), from individual galaxies at high redshift, the fields of view are very small, and the integration times are long. These telescopes are inadequate for measuring the galaxy distribution on the very large scales relevant to cosmological questions, such as the baryon acoustic oscillations at intermediate redshifts, or the large-scale distribution of galaxies that reionize the universe. The latter is of particular interest for cross correlation studies with very wide field, low-resolution $\mathrm{H} \mathrm{I} 21 \mathrm{~cm}$ images of the intergalactic medium during cosmic reionization (Lidz et al. 2011).
The integrated millimeter and submillimieter line emission from early galaxies has also been recognized as a possible significant contaminant of measurements of the spectral and spatial fluctuations of the cosmic microwave background (CMB; Righi et al. 2008a, 2008b; Chluba \& Sunyaev 2012; de Zotti et al. 2015; Mashian et al. 2016). For example, modeling suggests (Mashian et al. 2016) that the integrated CO line emission could be significantly higher than the primordial spectral distortions due to other cosmological effects (e.g., Chluba \& Sunyaev 2012; Sunyaev \& Khatri 2013; Tashiro 2014), and may be measurable with next generation instruments like the Primordial Inflation Explorer (PIXIE; Kogut et al. 2014). ${ }^{14}$

Numerous calculations have been done to predict the mean sky brightness due to emission lines from $\mathrm{CO}$ at intermediate and high redshift, and [C II] $158 \mu \mathrm{m}$ emission at very high redshift (see Section 2). These predictions are based on either

\footnotetext{
14 PIXIE is a space observatory concept to map the CMB over the frequency range $30 \mathrm{GHz}$ to $6 \mathrm{THz}$, one goal of which is to constrain the average CMB energy spectrum with much greater accuracy than FIRAS.
} 
empirical estimates using proxies for the line emission, such as the cosmic star formation rate density, or large scale cosmological simulations of galaxy formation, with recipes to relate proxy measurements or simulated properties to line luminosities.

In this paper, we present direct measurements of the summed line luminosity from individual sources in bands around 99 and $242 \mathrm{GHz}$. These measurements are based on the ALMA spectral deep field observations (ASPECS) program, corresponding to a broad band spectral line deep field of the UDF at 1.25 and $3 \mathrm{~mm}$ (Aravena et al. 2016a; Decarli et al. 2016a; Walter et al. 2016). From these measurement, we derive the mean brightness temperature at a given observing frequency due to high redshift galaxies. As a pilot study with ALMA, the fields are necessarily small, and the number of galaxies few. However, the measured quantity is direct: line emission from early galaxies. Hence, no modeling or conversion factors are required.

\section{MODEL PREDICTIONS FOR THE LINE BRIGHTNESS}

The dominant contribution to the integrated line brightness from high redshift galaxies in the $99 \mathrm{GHz}$ band is due to rotational transitions of $\mathrm{CO}$ from galaxies at intermediate to high redshift. Other molecular tracers, such as high dipole moment molecules like $\mathrm{HCN}$ and $\mathrm{HCO}^{+}$, are typically 10 times, or more, fainter than $\mathrm{CO}$, while the atomic fine structure lines would be from galaxies at improbable redshifts $(z \sim 20$; see Carilli \& Walter 2013). At $242 \mathrm{GHz}$, the integrated line brightness will be some combination of higher order $\mathrm{CO}$ lines from intermediate and high redshift galaxies, plus a possible contribution from [C II] $158 \mu \mathrm{m}$ line emission from galaxies at $z \sim 6$ to 7 , and other fine structure lines at lower redshift. We consider each in turn.

Considering CO in the $99 \mathrm{GHz}$ band, predictions of the mean CO sky brightness from early galaxies have taken two approaches. First is an empirical use of the measured evolution of the cosmic star formation rate density, and/or the cosmic far-infrared (FIR) background, converted to CO luminosity by adopting a CO-to-FIR or star formation rate conversion factor (Righi et al. 2008a, 2008b; Lidz et al. 2011; de Zotti et al. 2015). A related calculation is to consider the star formation rate density required to reionize the neutral intergalactic medium at high redshift, subsequently converted to $\mathrm{CO}$ luminosity using said conversion factors (Carilli 2011; Gong et al. 2011). While based on celestial measurements, these methods involve significant uncertainties inherent in both the determination of the cosmic star formation rate density, and more importantly, the assumed "star formation law" relating CO luminosity to FIR luminosity, or to star formation rate (Kennicutt \& Evans 2012; Carilli \& Walter 2013). The latter may entail a dual conversion of star formation rate to total gas mass, then total gas mass to CO luminosity. There is the additional uncertainty in the assumed gas excitation when modeling the contribution to the mean brightess at a given observing frequency from different $\mathrm{CO}$ transitions from galaxies at different redshifts.

The second method for predicting the mean CO sky brightness is through cosmological numerical simulations (Gong et al. 2011; Li et al. 2016; Mashian et al. 2016). Such simulations can be normalized to e.g., an observed galaxy luminosity function at a given redshift, although ultimately, even the most detailed simulations rely on recipes to convert from simulated to observable quantities. This is particularly difficult in the case of tracer molecules, such as $\mathrm{CO}$, while also including their excitation state.

In summary, the predictions at around $100 \mathrm{GHz}$ for the mean brightness from $\mathrm{CO}$ lines from intermediate and high redshift galaxies range from $1.5 \mu \mathrm{K}$ (Righi et al. 2008a, 2008b) to about $10 \mu \mathrm{K}$ (Marshian et al. 2016).

Two recent observations have set upper limits to the $\mathrm{CO}$ brightness from distant galaxies using $\mathrm{CO}$ intensity mapping. The first entailed a cross correlation of WMAP images with maps of very large-scale structures from the SDSS, namely the photometric quasar sample and the luminous red galaxy sample (Pullen et al. 2013). The cross correlation technique removes numerous systematic errors. Pullen et al. estimate upper limits to the mean brightness temperature of $\mathrm{CO} 1-0$ or 2-1 of about $10 \mu \mathrm{K}$ in the $30-90 \mathrm{GHz}$ range. The second was an interferometric measurement of the brightness fluctuations at $30 \mathrm{GHz}$ using the Sunyaev-Zel'dovich Array (Keating et al. 2015). Keating et al. quote an upper limit to the CO 1-0 mean brightness of $\sim 5 \mu \mathrm{K}$ from $z \sim 3$ galaxies. Since both these measurements rely on modeling of the spatial structure in the signal, they depend on the assumed underlying structural parameters.

Considering the $242 \mathrm{GHz}$ band, predictions also vary considerably. The most detailed modeling to date, including analysis of lines from $\mathrm{CO},[\mathrm{C} \mathrm{II}]$, and [C I], is presented in Yue et al. (2015). They use large scale cosmological simulations, plus physically motivated conversion factors (Pallottini et al. 2015; Vallini et al. 2015), to derive the line luminosities from early galaxies. They predict a [C II] $158 \mu \mathrm{m}$ brightness of $\sim 0.05 \mu \mathrm{K}$ around $242 \mathrm{GHz}$ from $z \sim 6.5$ galaxies. At this frequency, they obtain a similar contribution from the $[\mathrm{CI}]$ lines at rest frame frequencies of 492 and $809 \mathrm{GHz}$, from lower redshift galaxies. The dominant line contribution to the mean brightness at $242 \mathrm{GHz}$ in their models comes from $\mathrm{CO}$ emission from galaxies at intermediate to high redshift, for which they derive a mean brightness of $\sim 0.45 \mu \mathrm{K}$. Note that, in their models, the [C II] contribution increases rapidly with increasing observing frequency, to $\sim 0.4 \mu \mathrm{K}$ at $316 \mathrm{GHz}$ (comparable to $\mathrm{CO}$ ), due to galaxies at $z \sim 5$. Conversely, Gong et al. (2012) perform an analytic calculation of the expected [C II] surface brightness based on interstellar medium physics and halo statistics, and predict a substantially larger contribution of [C II] at $242 \mathrm{GHz}$ of $\sim 0.3 \mu \mathrm{K}$.

\section{ASPECS: A BLIND SEARCH FOR MILLIMETER LINE EMISSION FROM HIGH REDSHIFT GALAXIES}

The ASPECS results are described in Walter et al. (2016), Aravena et al. (2016a), Decarli et al. (2016a). In brief, we surveyed a $\sim 1 \operatorname{arcmin}^{2}$ field in the UDF to a 3- $\sigma$ depth of $\sim 0.05 \mathrm{Jy} \mathrm{km} \mathrm{s}^{-1}$ (assuming line widths of $200 \mathrm{~km} \mathrm{~s}^{-1}$ ) over the frequency range $84-115 \mathrm{GHz}(3 \mathrm{~mm})$, and of $\sim 0.13 \mathrm{Jy} \mathrm{km} \mathrm{s}^{-1}$ over the frequency range $212-272 \mathrm{GHz}$. In the analysis below, we adopt the mean frequencies for each band, which are 99 and $242 \mathrm{GHz}$.

The observations are sensitive to galaxies over a wide range in redshift, depending on $\mathrm{CO}$ transition. The typical $\mathrm{CO}$ luminosity limits are $\sim 2 \times 10^{9} \mathrm{~K} \mathrm{~km} \mathrm{~s}^{-1} \mathrm{pc}^{2}$ at $3 \mathrm{~mm}$ and $6 \times$ $10^{8} \mathrm{~K} \mathrm{~km} \mathrm{~s}^{-1} \mathrm{pc}^{2}$ at $1 \mathrm{~mm}$ at $1<z<3$. The implied gas mass limits will depend on which CO transition is being considered, at which redshift, and depend critically on assumed $\mathrm{CO}$ excitation, in particular for the high order transitions, since the 
total gas mass is derived by extrapolation to low order. For reference, at the typical redshifts and transitions of the detected galaxies, these limits imply galaxies with gas masses of 3 to $10 \times 10^{9} M_{\odot}$, for a Galactic conversion factor of $\mathrm{CO} 1-0$ luminosity to total molecular gas mass. We discuss this point further in Section 5.1. For [C II] at very high redshift $(z \sim 6.5)$, our observations are sensitive to galaxies with star formation rates $\geqslant 10 M_{\odot} \mathrm{yr}^{-1}$, using the de Looze et al. (2011) conversion.

Line emitting galaxies were identified using multiple threedimenional search algorithms, and a series of tests were made for completeness and fidelity (Walter et al. 2016). Once a line candidate was identified, a search was made for an optical or near-IR (NIR) counterpart. Decarli et al. (2016a) discuss how a given line is identified as a specific $\mathrm{CO}$ transition. In some cases, the detection (or the lack of detection) of multiple $\mathrm{CO}$ transitions over the broad frequency range constrains the redshift determination. If an optical/NIR counterpart is present, literature information on the redshift of the source (via spectroscopy or SED fitting of the photometry) was also used. The lack of an optical counterpart is used to rule out low redshift interpretations in some cases. Ultimately, for some sources there can be ambiguity as to the transition in question, and therefore the redshift. This is dealt with via a bootstrapping approach (see Decarli et al. 2016a). However, in the context of the analysis below, this is not an issue, since we simply sum all the lines detected in the blind survey in a give observing band, independent of what transition and redshift the line happens to be.

For completeness, Figure 1 shows a compilation of all the candidate line detections in the survey, as presented in Walter et al. (2016). There are a total of 21 candidate lines above $5 \sigma$. In six cases, line identifications are unequivocally confirmed, through detection of other $\mathrm{CO}$ transitions, and/or an optical galaxy with a spectroscopic redshift. For the rest of the lines, extensive quantitative tests are made, and we only include lines with a $>60 \%$ "fidelity" rating. See Decarli et al. (2016a) for more details on the statistical analysis, and Walter et al. (2016) for total intensity $\mathrm{CO}$ images, and optical images, of all the candidates. At this fidelilty level, we expect to have roughly as many spurious detections as sources missing from the survey due to noise fluctuations (Decarli et al. 2016a).

\section{MEAN BRIGHTNESS TEMPERATURES}

The mean line brightness temperatures are calculated using an empirical approach of summing the lines detected in the blind survey. The process is simple. Table 2 in Walter et al. (2016) lists the lines detected according to the blind search criteria outlined in Section 3. We sum the total flux from all the lines detected in a given band, in $\mathrm{Jy} \mathrm{km} \mathrm{s}^{-1}$, which is equivalent dimensionally to $\mathrm{Jy} \mathrm{Hz}$ or $\mathrm{erg} \mathrm{s}^{-1} \mathrm{~cm}^{-2}$. We then divide by the total bandwidth covered in the blind survey, which results in a mean flux density over the full band and over the full field, $S_{\nu}$, in Jy. The mean brightness temperature at the observed wavelength, $\lambda_{\text {obs }}$, is then derived using the angular area of the field employed in the blind search, $\Omega_{f}$, under the Rayleigh-Jeans appoximation: $\quad T_{B} \sim 1360 S_{\nu} \lambda_{\text {obs }}^{2} \Omega_{f}^{-1} \mathrm{~K}$, where $\lambda_{\text {obs }}$ is in centimeters and $\Omega_{f}$ is in $\operatorname{arcsec}^{2}$.

For the $99 \mathrm{GHz}$ band, the total flux for the 10 lines detected in the band is $2.53 \pm 0.25 \mathrm{Jy} \mathrm{km} \mathrm{s}^{-1}=(8.3 \pm 0.08) \times 10^{5} \mathrm{Jy} \mathrm{Hz}$. The total bandwidth is $31 \mathrm{GHz}$, so the mean flux density across the band is $S_{\nu}=(2.7 \pm 0.27) \times 10^{-5} \mathrm{Jy}$. The field covered by the survey was $3600 \operatorname{arcsec}^{2}$. Hence, the mean brightness temperature, $T_{B}=0.94 \pm 0.09 \mu \mathrm{K}$.

For the $242 \mathrm{GHz}$ band, the total flux for the 11 lines detected in the band is $6.93 \pm 0.42 \mathrm{Jy} \mathrm{km} \mathrm{s} \mathrm{s}^{-1}=(5.6 \pm 0.34)$ $\times 10^{6} \mathrm{Jy} \mathrm{Hz}$. The total bandwidth is $60 \mathrm{GHz}$, so the mean flux density across the band is $S_{\nu}=(9.3 \pm 0.6) \times 10^{-5} \mathrm{Jy}$. The field covered by the survey was also $3600 \operatorname{arcsec}^{2}$. Hence, the mean brightness temperature, $T_{B}=0.55 \pm 0.033 \mu \mathrm{K}$.

\section{DISCUSSION}

\subsection{Limits}

As a pilot ALMA study, we reemphasize that the volumes in question are small, as are the number of detections. Hence, our conclusions and uncertainties are dominated by cosmic variance and simple shot noise (Poisson statistics). Aravena et al. (2016b) consider the issue of cosmic variance in the context of our particular field. Based on the drop-out galaxy counts, and the bright sub-mm source counts, this bias might be as large as a factor two (low). On the other hand, consideration of the contribution of faint sub-mm continuum sources to the cosmic IR background, based on our deeper ASPECS ALMA data, suggests a factor closer to unity (Aravena et al. 2016b). Regardless, since this is a direct survey of the observable in question, namely, mean brightness due to line emission from distant galaxies at a given observing frequency, the results remain of interest in general progress toward millimeter line intensity mapping, and a factor two uncertainty is inconsequential for our analysis in Section 5.2.

Our measurements are also lower limits, since we only sum lines detected. We do not extrapolate to, e.g., lower or higher luminosity galaxies using an assumed luminosity function. Considering CO (the dominant contributor at $99 \mathrm{GHz}$, certainly, and likely at 242 as well), our detection threshold was set in order to reach what may be the "knee" in the CO luminosity function at the primary redshifts to which our survey is most sensitive $(z \sim 1-3)$. This estimation was based on both numerical simulations and extrapolations of $\mathrm{CO}$ emission properties of high redshift galaxies from, e.g., measures of dust luminosities or star formation rates (see Decarli et al. 2016a for more details). If the CO luminosity function is relatively flat at low luminosities, and steep at high luminosities, then galaxies around the knee of the curve dominate the overall luminosity. For example, using the Popping et al. (2016a) and Lagos et al. (2012) CO luminosity functions and our limits at $99 \mathrm{GHz}$, we estimate that we should be detecting between $40 \%$ and $70 \%$ of the total CO luminosity (Decarli et al. 2016a) in this dominant redshift range.

\subsection{Comparison to Predictions and CMB Spectral Distortions}

As stated in Section 1, millimeter line intensity mapping experiments will have broad impact, from studies of galaxy formation to the baryon acoustic oscillations. In this section we consider in some detail our results in the context of one topical area that has seen considerable attention recently, namely, spectral distortions of the CMB.

In Section 2, we reviewed the predictions for the line brightness at 99 and $242 \mathrm{GHz}$ based on phenomenological calculations using proxies for the line luminosity (such as the cosmic star formation density), or numerical simulations of galaxy formation. Predictions vary significantly, but range from $\sim 1 \mu \mathrm{K}$ to $10 \mu \mathrm{K}$, in the frequency ranges being considered. To 

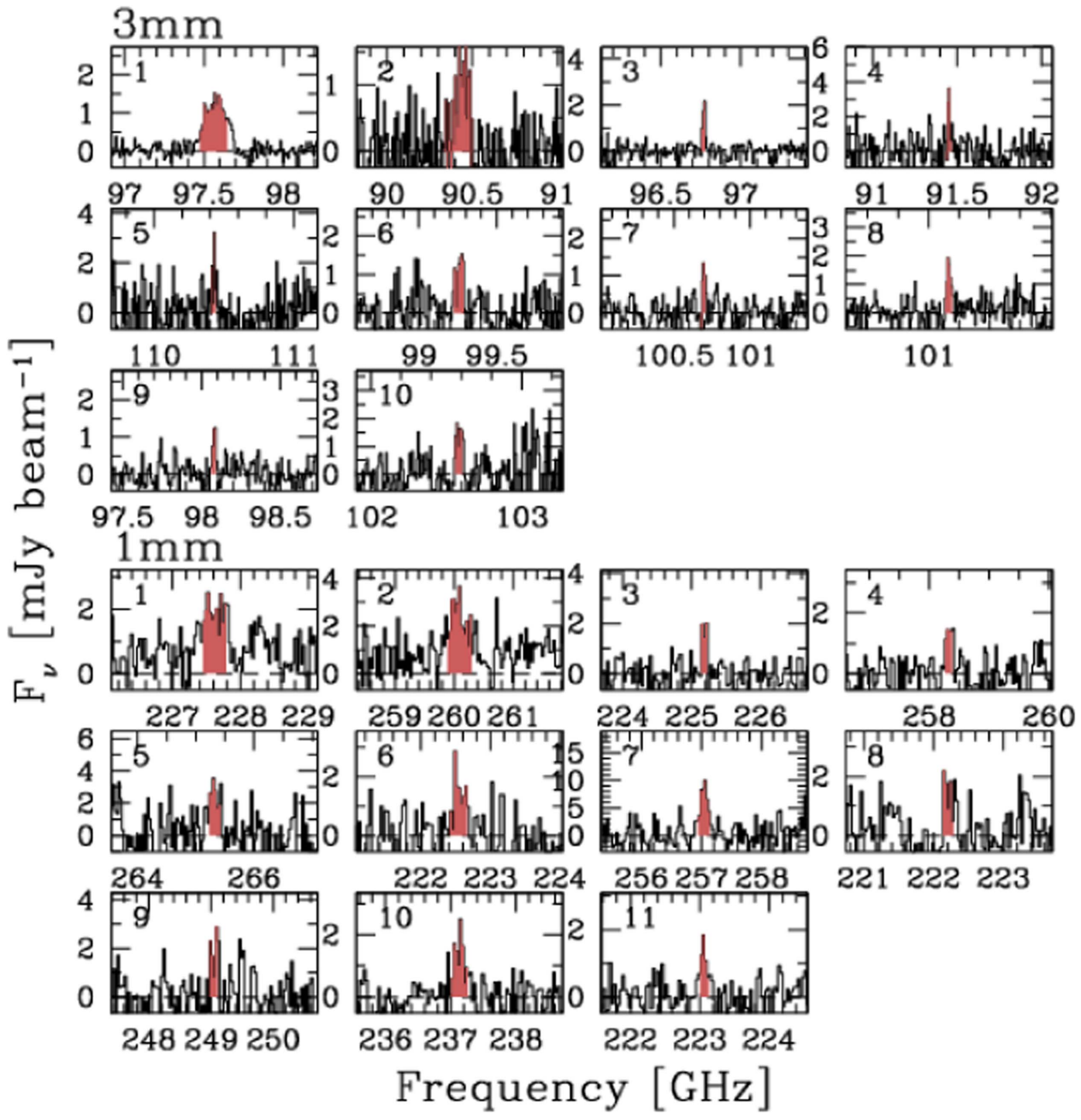

Figure 1. Montage of all the $>5 \sigma$ line candidates from the ASPECS blind survey (Walter et al. 2016). The red areas show the spectral region over which the signal-tonoise was calculated (see Decarli et al. 2016a).

within the uncertainties inherent in small volume surveys, our direct measurements of $T_{B}=0.94 \pm 0.09 \mu \mathrm{K}$ at $99 \mathrm{GHz}$ and $T_{B}=0.55 \pm 0.033 \mu \mathrm{K}$ at $242 \mathrm{GHz}$, argue for the faint end of these predictions, although we again emphasize that these should be treated as lower limits.

How do our measurements then compare to, for instance, the expected distortions in the CMB spectrum due to early energy release, and to the expected sensitivity of planned CMB spectral distortion experiments? As a benchmark for experimental sensitivity, we adopt the current parameters being considered for PIXIE (Kogut et al. 2011, 2014), using the $15 \mathrm{GHz}$ spectral resolution for the proposed experiment. Considering the expected sky brightness contributions, we focus on the more cosmologically relevant predictions, relating to recombination and reionization.

We note that there are other potentially significant foregrounds, in particular, Galactic and extragalactic thermal emission from warm dust, and synchrotron emission. Kogut et al. (2014) review the relative magnitudes of these contributions. The thermal emission from warm dust, in particular, is calculated to be an order of magnitude, or more, stronger than the summed millimeter line emission considered herein. However, the spectral behavior of the dust emission is considered to be well understood, and should be well modeled, and removed, using spectral fitting algorithms over a broad frequency range. Herein, we focus on the millimeter and submillimeter line emission, given that this is our measured quantity, and compare it to the predicted cosmological signals. Additional discussion of foregrounds can be found in, e.g., de Zotti et al. (2015).

In Figure 2, we show a comparison of various distortion signals, along with the line limits derived herein. We focus on guaranteed distortions within $\Lambda$ CDM (see Chluba 2016, for most recent overview), some of which should be detectable with future experiments, at least in terms of raw sensitivity (Kogut et al. 2011). A wider range of energy release processes (e.g., decaying particle scenarios) is discussed in Chluba (2013) and Chluba \& Jeong (2014).

The largest $\mathrm{CMB}$ expected spectral distortion is created at low redshift by the reionization and structure formation process (Sunyaev \& Zeldovich 1972; Hu et al. 1994a). This signal is close to a pure Compton-y distortion (Zeldovich \& Sunyaev 1969) caused through partial up-scattering of CMB blackbody photons by hot electrons yielding a $y$-parameter $y \simeq 2 \times 10^{-6}$ (e.g., Refregier et al. 2000; Hill et al. 2015). Contributions from the hot gas $(\simeq 1 \mathrm{keV})$ residing in low mass halos also give rise to a noticeable relativistic temperature correction, which could be used to constrain the average temperature of baryons at low redshifts (Hill et al. 2015). While the relativistic correction signal requires removal of the integrated $\mathrm{CO}$ emission, the non- 


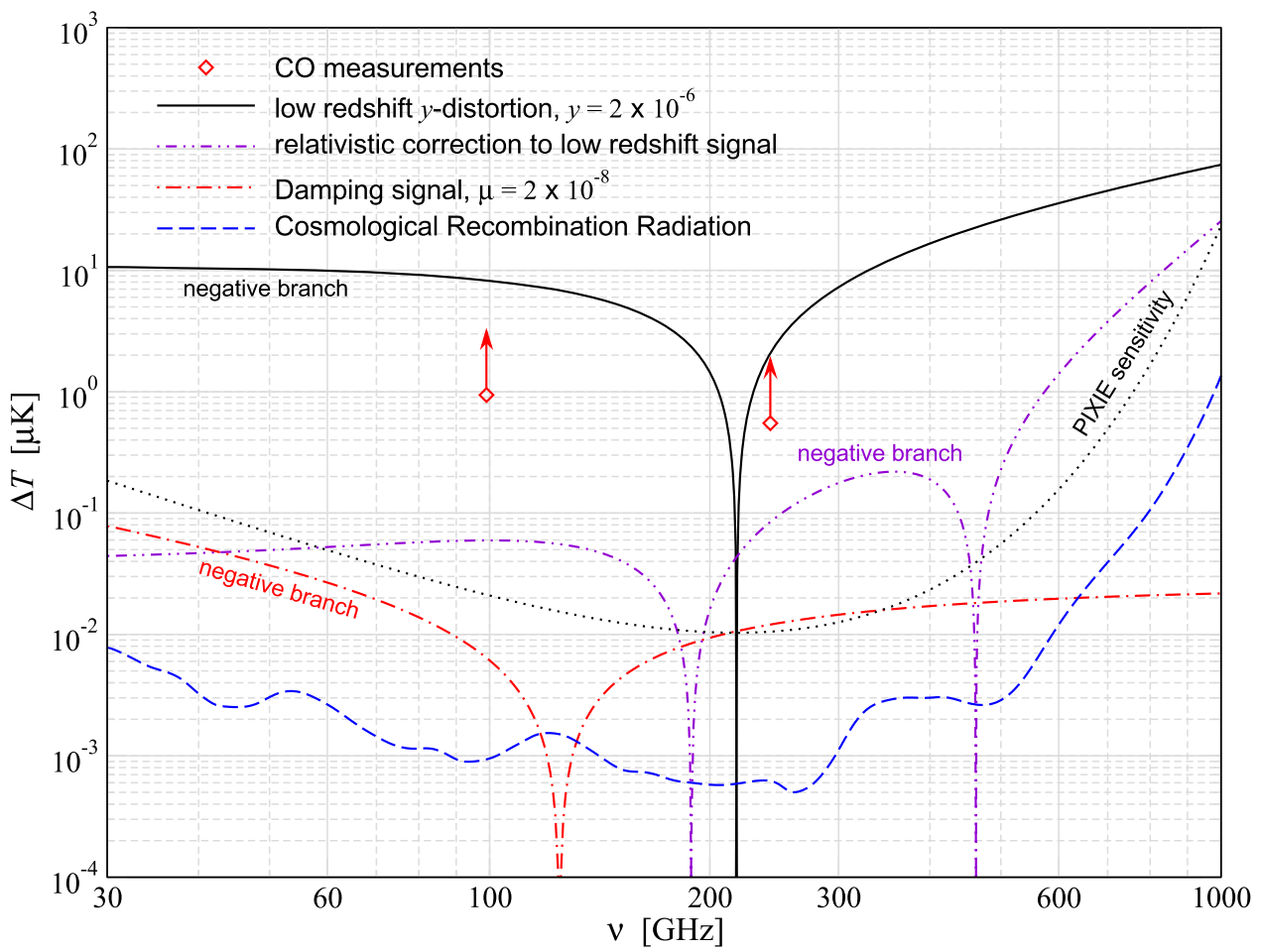

Figure 2. Comparison of different CMB distortion signals (negative branches of the signals are marked) with the millimeter line limits reported in this paper. The lowredshift distortion created by reionization and structure formation is close to a $y$-distortion with $y \simeq 2 \times 10^{-6}$. Contributions from the hot gas in low mass halos give rise to a noticeable relativistic temperature correction. For the damping signal, we plot a $\mu$-distortion with $\mu=2 \times 10^{-8}$. The cosmological recombination radiation was computed using CosmoSpec. The estimated effective sensitivity $\left(\Delta I_{\nu} \approx 5 \mathrm{Jy} \mathrm{sr}^{-1}\right)$ of PIXIE is shown for comparison (dotted line).

relativistic $y$-distortion contribution should be less affected and separable using multi-frequency capabilities of future experiments.

Another inevitable distortion is created by the dissipation of small-scale fluctuations in the primordial photon-baryon plasma (Sunyaev \& Zeldovich 1970; Daly 1991; Hu et al. 1994b; Chluba et al. 2012b) due to Silk damping. We illustrate the $\mu$-distortion (Sunyaev \& Zeldovich 1970) contribution of this signal using $\mu=2 \times 10^{-8}$, which is close to the value expected for the $\Lambda$ CDM cosmology (Chluba 2016). A $\mu$ distortion can only be created in dense and hot environments present in the early universe at $z \gtrsim 5 \times 10^{4}$ (Burigana et al. 1991; Hu \& Silk 1993). By detecting this signal one can probe the amplitude of perturbations at scales far smaller than those seen in the CMB anisotropies, delivering another independent way to test different inflation models (e.g., Chluba et al. 2012a, 2012b; Dent et al. 2012; Clesse et al. 2014).

Finally, we show the cosmological hydrogen and helium recombination radiation emitted at $z \simeq 10^{3}$ (Peebles 1968; Zeldovich et al. 1968; Dubrovich 1975; Kholupenko et al. 2005; Chluba \& Sunyaev 2006; Rubiño-Martín et al. 2006), which was computed using CosmoSpec (Chluba \& AliHaïmoud 2016). This signal could provide an independent way to constrain cosmological parameters and directly map the recombination history (Sunyaev \& Chluba 2009). It is unpolarized and its unique spectral variability is very hard to mimic by other foregrounds or instrumental effects. ${ }^{15}$

15 The expected distortions due to annihilating dark matter (McDonald et al. 2001; Chluba 2010; Chluba \& Sunyaev 2012; Chluba 2013) and the differences in the cooling of baryons relative to $\mathrm{CMB}$ photons during cosmic expansion (Chluba 2005; Chluba \& Sunyaev 2012) were not illustrated here.
The latter two effects cause fractional spectral distortions in the range of $10^{-9}-10^{-8}$, implying observed brightness temperature perturbations $\Delta T_{\mathrm{B}} \simeq 3 \mathrm{nK}-30 \mathrm{nK}$, well below the contribution of the mean line brightness measured herein. Thus, beyond doubt, extraction of these primordial distortions will be very challenging, requiring sophisticated foreground removal techniques, unprecedented control of systematics, broad spectral coverage, and high sensitivity multi-frequency capabilities. To successfully remove the integrated millimeter and submillimeter line emission, it will be advantageous to exploit the synergies between future CMB distortion measurements and observations similar to those presented here. Given the importance of the primordial distortion signals to studies of early-universe physics, this direction is highly relevant.

As ALMA attains full capability, spectral deep fields will become more efficient and effective, eventually encompassing areas of tens of square arcminutes. Our pilot studies have already shown the impact of such measurements over a broad range of problems in modern astrophysics and cosmology. In parallel, the Jansky Very Large Array is exploring similar deep spectral searches at $30 \mathrm{GHz}$ (e.g., Lentati et al. 2015; D. Riechers et al. 2016, in preparation), while the advent of high frequency spectral cameras on the Green Bank Telescope provides a sensitive platform for wide field spectral searches (Sieth et al. 2016). In the long term, a "Next Generation Very Large Array," operating between 20 and $115 \mathrm{GHz}$ with octave, or broader, bandwidth receivers and 10 times the collecting area of ALMA and the JVLA, has the potential to revolutionize blind searches for molecular gas in the early universe (Carilli et al. 2015; Casey et al. 2015).

We thank the referee for useful comments that improved the paper. FW acknowledges support through ERC grant COSMIC- 
DAWN. MA acknowledges partial support from FONDECYT through grant 1140099 . Support for RD was provided by the DFG priority program 1573 "The physics of the interstellar medium." DR acknowledges support from the National Science Foundation under grant number AST-\#1614213 to Cornell University. FB acknowledges support by the Collaborative Research Council 956, sub-project A1, funded by the Deutsche Forschungsgemeinschaft (DFG). This paper makes use of the following ALMA data: ADS/JAO.ALMA\#2013.1.00146.S and ADS/JAO.ALMA\#2013.1.00718.S. ALMA is a partnership of ESO (representing its member states), NSF (USA), and NINS (Japan), together with NRC (Canada), NSC and ASIAA (Taiwan), and KASI (Republic of Korea), in cooperation with the Republic of Chile. The Joint ALMA Observatory is operated by ESO, AUI/NRAO, and NAOJ. The National Radio Astronomy Observatory is a facility of the National Science Foundation operated under cooperative agreement by Associated Universities, Inc.

\section{REFERENCES}

Aravena, M., Decarli, R., Walter, F., et al. 2016a, ApJ, 833, 68 (Paper II) Aravena, M., Decarli, R., Walter, F., et al. 2016b, ApJ, 833, 71 (Paper V) Burigana, C., Danese, L., \& de Zotti, G. 1991, A\&A, 246, 49

Carilli, C. 2011, ApJL, 730, L30

Carilli, C. L., McKinnon, M., Ott, J., et al. 2015, arXiv:1510.06438

Carilli, C. L., \& Walter, F. 2013, ARA\&A, 51, 105

Casey, C., Hodge, J., Lacy, M., et al. 2015, arXiv:1510.06411

Chluba, J. 2005, PhD thesis, Ludwig Maximilians Univ.

Chluba, J. 2010, MNRAS, 402, 1195

Chluba, J. 2013, MNRAS, 436, 2232

Chluba, J. 2016, MNRAS, 460, 227

Chluba, J., Erickcek, A. L., \& Ben-Dayan, I. 2012a, ApJ, 758, 76

Chluba, J., \& Jeong, D. 2014, MNRAS, 438, 2065

Chluba, J., Khatri, R., \& Sunyaev, R. 2012b, MNRAS, 425, 1129

Chluba, J., \& Sunyaev, R. 2012, MNRAS, 419, 1294

Clesse, S., Garbrecht, B., \& Zhu, Y. 2014, JCAP, 10, 046

Daly, R. A. 1991, ApJ, 371, 14

de Looze, I., Baes, M., Bendo, G., Cortese, L., \& Fritz, J. 2011, MNRAS, 416,2712 de Zotti, G., Negrello, M., Castex, G., Lapi, A., \& Bonato, M. 2015, arXiv: 1512.04816

Decarli, R., Walter, F., Aravena, M., et al. 2016a, ApJ, 833, 69 (Paper III)

Dent, J. B., Easson, D. A., \& Tashiro, H. 2012, PhRvD, 86, 023514

Dubrovich, V. K. 1975, SvAL, 1, 196

Gong, Y., Cooray, A., Silva, M. B., et al. 2011, ApJL, 728, L46

Gong, Y., Cooray, A., Silva, M. B., et al. 2012, ApJ, 745, 49

Hill, J. C., Battaglia, N., Chluba, J., et al. 2015, PhRvL, 115, 261301

Hu, W., Scott, D., \& Silk, J. 1994a, ApJL, 430, L5

Hu, W., Scott, D., \& Silk, J. 1994b, PhRvD, 49, 648

Hu, W., \& Silk, J. 1993, PhRvD, 48, 485

Keating, G., Bower, G., Marrone, D., et al. 2015, ApJ, 814, 140

Kennicutt, R., \& Evans, N. 2012, ARA\&A, 50, 531

Kholupenko, E. E., Ivanchik, A. V., \& Varshalovich, D. A. 2005, GrCo, 11,161

Kogut, A., Chuss, D., Dotson, J., et al. 2014, SPIE, 9143, ID91431E

Kogut, A., Fixsen, D. J., Chuss, D., et al. 2011, JCAP, 7, 25

Lagos, C., Bayet, E., Baugh, C., et al. 2012, MNRAS, 426, 2142

Lentati, L., Wagg, J., Carilli, C., et al. 2015, ApJ, 800, 67

Li, T. Y., Wechsler, R., Devaraj, K., \& Church, S. 2016, ApJ, 817, 169

Lidz, A., Furlanetto, S., Oh, S.-P., et al. 2011, ApJ, 741, 70

Mashian, N., Loeb, A., \& Sternberg, A. 2016, MNRAS, 458, L99

McDonald, P., Scherrer, R. J., \& Walker, T. P. 2001, PhRvD, 63, 023001

Pallottini, A., Ferrara, A., Pacucci, F., et al. 2015, MNRAS, 453, 2465

Peebles, P. J. E. 1968, ApJ, 153, 1

Popping, G., van Kampen, E., Decarli, R., et al. 2016, MNRAS, 461, 93

Pullen, A., Chang, Z., Dore, O., \& Lidz, A. 2013, ApJ, 768, 15

Refregier, A., Komatsu, E., Spergel, D. N., \& Pen, U.-L. 2000, PhRvD, 61, 123001

Righi, M., Hernández-Monteagudo, C., \& Sunyaev, R. A. 2008a, A\&A, 478,685

Righi, M., Hernandez-Monteagudo, C., \& Sunyaev, R. A. 2008b, A\&A, 498, 489

Rubiño-Martín, J. A., Chluba, J., \& Sunyaev, R. A. 2006, MNRAS, 371, 1939

Sunyaev, R. A., \& Chluba, J. 2009, AN, 330, 657

Sunyaev, R. A., \& Khatri, R. 2013, IJMPD, 22, 1330014

Sunyaev, R. A., \& Zeldovich, Y. B. 1970, Ap\&SS, 7, 20

Sunyaev, R. A., \& Zeldovich, Y. B. 1972, A\&A, 20, 189

Tashiro, H. 2014, PTEP, 2014, 06B107

Vallini, L., Gallerini, S., Ferrara, A., et al. 2015, ApJ, 813, 36

Walter, F., Decarli, R., Aravena, M., et al. 2016, ApJ, 833, 67 (Paper I)

Yue, B., Ferrara, A., Pallottini, A., Gallerani, S., \& Vallini, L. 2015, MNRAS, 450, 3829

Zeldovich, Y. B., Kurt, V. G., \& Sunyaev, R. A. 1968, ZhETF, 55, 278

Zeldovich, Y. B., \& Sunyaev, R. A. 1969, Ap\&SS, 4, 301 